Published in final edited form as:

J Am Dent Assoc. 2009 September ; 140(Suppl 1): 44S-48S.

\title{
A view of the future:
}

\section{Dentistry and oral health in America}

\author{
Dr. Isabel Garcia, DDS, MPH [Deputy director] and \\ National Institute of Dental and Craniofacial Research, National Institutes of Health, 31 Center \\ Drive, Building 31, Room 2C39, Bethesda, Md. 20892 \\ Dr. Lawrence A. Tabak, DDS, PhD [Director] \\ National Institute of Dental and Craniofacial Research, National Institutes of Health, Bethesda, \\ Md
}

Isabel Garcia: Garcial@mail.nih.gov

\begin{abstract}
Background-New tools are enabling researchers to understand the mysteries of oral biology and disease and to change profoundly the treatment of oral, dental and craniofacial diseases and disorders. The authors explore advances in modern science and technology and how they will change oral health care in the future.
\end{abstract}

Results-Emerging technologies such as salivary diagnostics, high-resolution imaging and nanotechnologies, as well as other new tools will lead to efficient and highly effective personalized dental treatments. A new generation of cell-based therapies will be available for regenerating tissues, and anti-inflammatory drugs and pain medications will be tailored to maximize efficacy and safety. Large teams of clinicians and scientists will tackle increasingly complex problems, and advances in computational sciences will make it possible to create virtual teams across the world. Information technology systems will enable clinicians to examine and integrate information obtained from all databases in cyberspace.

Clinical Implications-As scientists discover newer and better methods to preempt and prevent disease, they must translate these methods into tools for people at greatest risk of developing disease. Conquering the array of complex diseases that affect the oral and craniofacial complex will require multifaceted strategies and multidisciplinary cooperation.

\section{Keywords}

Dental research; emerging technology

American social writer and philosopher Eric Hoffer wrote, "In times of drastic change, the learners inherit the future. The learned usually find themselves beautifully equipped to live in a world that no longer exists."1

More than 60 years ago, the National Institute of Dental and Craniofacial Research (NIDCR) was created to ensure the dental health of people in the United States. Since then, the world of research has undergone such drastic changes that, scientifically, the 1940s seem

Copyright @ 2012 American Dental Association. All rights reserved.

Address reprint requests to Dr. Garcia

Disclosure. Drs. Garcia and Tabak did not report any disclosures. 
as distant as medieval times. Our investments in research have improved the lives of millions of people and demonstrated that oral health is integral to overall health.

Revolutionary tools unimaginable even a decade ago now are enabling researchers to unravel the mysteries of oral biology, oral health and disease. Genomic and proteomic advances combined with the power of super-fast computers are profoundly changing our understanding of oral, dental and craniofacial diseases and disorders. More precise and faster diagnostic tests, new drugs and biologics, practice-based research and culturally sensitive interventions are providing novel avenues to improve oral health. As more remarkable discoveries and greater achievements in science and technology emerge, Hoffer's words give us an irresistible mandate to embrace lifelong learning.

\section{THE PERILS OF PREDICTIONS}

Predicting the future is risky business. In 1977, Ken Olsen, the president and founder of Digital Equipment Corporation (headquartered from 1957 to 1992 in Maynard, Mass.), maker of large business mainframe computers, is said to have argued against personal computers, stating "there is no reason for any individual to have a computer in [his] home."2 Although the statement was not in reference to the modern personal computer, the perceived error in judgment reportedly played a large role in the company's demise. ${ }^{3}$

French surgeon Dr. Alfred Velpeau offered a downbeat view of the future. In 1839, he declared, "The abolishment of pain in surgery is a chimera. It is absurd to go on seeking it today. 'Knife' and 'pain' are two words in surgery that must forever be associated in the consciousness of the patient." ${ }^{4}$ Fortunately, for both patients and surgeons, this pronouncement was wrong. Another example of the perils of forecasting the future came from surgeon Pierre Pachet, who, in 1872, said that "Louis Pasteur's theory of germs is ridiculous fiction." ${ }^{.5}$ Finally, in the oral health arena, the forecasting of a severe shortage of dentists in the United States in the mid-1960s was never realized as a result of unforeseen sharp declines in population growth and the increased federal support for dental schools. ${ }^{6}$

According to American theoretical physicist Michio Kaku, "We are making the historic transition from the age of scientific discovery to the age of scientific mastery in which we will be able to manipulate and mould nature almost to our wishes."7 Regardless of whether we achieve the kind of scientific and technological utopia described by Kaku, it is reasonable to predict that within the next two decades, science will give us the tools to shortcircuit diseases at the molecular level, to alter the course of disorders by manipulating defective genes, and to treat conditions by using drugs or other therapies customized with stealth precision for individual patients.

The visual and tactile methods of dental diagnostics will be augmented by powerful technologies such as smart imaging systems, genome scans to evaluate patients' responses to pharmaceuticals, molecularly based diagnostics and integrated electronic risk management systems. Dentists of the future will rely on a range of diagnostic and treatment tools that rapidly and efficiently process a patient's biological information, from their genes to their proteins to their metabolites.

\section{DENTISTRY IN THE FUTURE}

Looking beyond the horizon, what changes can we predict? Can we achieve a future in which prevention and delivery of care empower all Americans to maintain good oral health for a lifetime? Can we develop smart, early interventions to avert suffering from chronic orofacial pain or craniofacial disorders? Will we have the tools to allow clinicians to recognize oral malignancies at their earliest stage and short-circuit their progress? Will we 
successfully bioengineer replacement teeth or discover small molecules to alter the composition of oral biofilms? Can the salivary glands be a gateway to the body for the delivery of precise molecular therapies with few side effects?

Dental research is well-positioned to attain all of these goals. However, our success will depend not only on the remarkable advances of modern science and technology, but also on our ability to achieve closer integration between dental research, dental practice and education. Conquering the array of complex diseases that affect the oral and craniofacial complex will require multifaceted strategies and multidisciplinary cooperation, not professional isolationism.

\section{Predictive tools}

One area of science that holds exceptional potential to alter the practice of dentistry is salivary diagnostics. This emerging technology will allow dentists to make a quantum leap forward in their ability to predict, detect and prevent disease. Consider the following scenario:

On entering a dental office, a new patient will expectorate into a small vial. A staff member will load the contents onto a disposable diagnostic chip about the size of a dime. This all-inone chip first will isolate the patient's DNA and rapidly decode the genetic blueprint of life. This information will be analyzed for drug response genes, and a staff member will add automated alerts to the patient's electronic record to ensure that the proper medication dosage will be provided if needed in the future.

This "lab on a chip" then will scan the saliva to determine the levels of numerous molecules present in the fluid; within minutes, a detailed report will be produced. Using specialized software and a computer, the clinician then will compare unusual changes in various proteins, antibodies or other analytes. He or she will use these results as the baseline measures against which findings from future visits will be compared. These tests will enable the early detection of various oral diseases, as well as reveal signs of developing medical conditions, ranging from cancer to diabetes to various infectious diseases (see illustration ${ }^{8}$ ).

\section{Personalized treatment}

In the dental operatory, patients likely will never see or hear a hand-piece. Dentists will perform routine examinations that include use of a high-resolution imaging device to better visualize the subsurface tomography of each tooth. In places where the enamel shows early signs of demineralization, the process will be reversed with advanced nanomaterials that deliver biologically based therapies to promote remineralization naturally. In cases of chronic periodontal disease, dentists will possess additional predictive tools to characterize the bacteria underlying the infection and the specific nature of the immune response, and they will be able to personalize treatments that most effectively target and eliminate both the bacteria and the infection. In the rare instances in which the disease persists and destroys tooth-supporting bone, dentists will know how to regenerate bone and prevent tooth loss.

\section{Targeted approaches}

Dentists also will possess high-resolution imaging devices to visualize any unusual lesions in the oral cavity. Pervasive computing, in the form of nanochips that can be implanted in the mouth or introduced into the circulation or soft tissues, will act as stealth sentinels yielding real-time information about the patient's health status. They will have an array of tools available to perform rapid molecular pathological analysis to help identify premalignant lesions and take the preemptive steps of characterizing the internal molecular patterns of these cells (much like whorls in a fingerprint) and matching them with a drug 
that kills the tumor cells selectively, preserving both healthy tissue and function. To reduce the likelihood of a recurrence, surgeons will have sophisticated imaging tools and stains at their disposal that indicate whether other tumor cells have spread inconspicuously nearby. Following this fluorescent trail, they will surgically remove all of the potentially affected tissues.

To treat people with oral autoimmune diseases, either localized or part of a complex syndrome, health care professionals will possess much more sensitive and precise blood- or saliva-based diagnostic tests. These tests will measure levels of disease-linked antibodies or defense cells as they begin to amass, but before overt symptoms occur. They will complement gene-scanning tests that alert a dentist or physician that his or her patient has an inherited susceptibility to diseases such as Sjögren syndrome or diabetes.

\section{Novel biologics and drugs}

People who have disfiguring wounds to the head and face or those who are born with birth defects will benefit from a new generation of cell-based therapies to regenerate tissues and heal wounds without common complications such as scarring, contracture or dysfunction. People with inflammatory diseases will benefit from a new generation of anti-inflammatory drugs that will enhance resolution of the immune response via natural signals to heal and cease the inflammation, rather than via an attempt to interrupt the immune response. In addition, the large number of people with chronic orofacial pain conditions will be treated with a new generation of non-addictive pain medications that will be tailored for them through the use of pharmacogenomic principles to maximize efficacy and safety while avoiding dangerous side effects.

\section{NEW WAYS OF CONDUCTING SCIENTIFIC RESEARCH}

Early science largely was a cottage industry. By the 1950s, investigators began forming small teams (for example, Watson, Crick and Franklin) to tackle complex problems requiring different sets of expertise. The 21 st century already has seen the self-assembly of large teams of clinicians and scientists to tackle increasingly complex problems, such as decoding the human genome. Extraordinary advances in computational sciences, such as cloud computing, have made it possible to create virtual teams that span the planet. ${ }^{9}$ The availability of massively scalable information technology systems will enable clinicians of the future to investigate and then integrate information obtained from all databases in cyberspace. Ironically, these new approaches will make it easier for the solo practitioner, who may be physically alone but who will be linked electronically with thousands of people comprising the scientific community worldwide.

Consider the situation in which one's patient has an unknown disease in the mouth. The clinician will analyze the clinical and laboratory findings and compare them with anonymized electronic records throughout the globe. He or she then will link the findings with those of extensive genetic studies of every type, as well as with the latest findings from clinical trials around the world. The clinician will use software to integrate and help interpret the blizzard of data at his or her disposal. In the end, predictive algorithms will provide the clinician with the seminal information needed to deduce a diagnosis and establish a course of action.

\section{SHARING THE HEALTH}

Science changes our lives. Today, we are on the verge of many opportunities to develop tailored, preemptive oral health care that will improve the health of and quality of life for millions of people. We are embracing new tools and technologies and applying new 
biologically based approaches to solving old problems. But we must be mindful that science has not reached everyone adequately. Stubborn inequalities in oral health continue to exist among many groups, communities, cities and towns. With respect to health disparities in the United States, it has been said, "Thus, it is not given in our genome that there must be inequalities in health. It is a social fact that most risk factors for common diseases, and many uncommon diseases, are more prevalent among the poor than among the wealthy."10

Dentistry can proudly claim a strong record of championing health promotion and disease prevention strategies, but many of our most effective preventive measures and messages have never reached those who need them most. As we discover and adopt newer and better methods to preempt and prevent disease, we must improve our ability to translate and disseminate these methods effectively into tools for communities and people at greatest risk of developing disease, or we will risk replicating the shortcomings of the past.

How do we turn the corner on health inequalities? Improving our understanding of what causes inequalities at individual, community and societal levels is necessary but not sufficient. Teasing out the underlying biological, behavioral and/or cultural factors is necessary but not sufficient. Targeted programs and interventions addressing unique populations are necessary but not sufficient.

Systematic action is needed to address oral health inequalities in a rational, purposeful and science-driven way that recognizes both societal and personal responsibility for health. Science and technology can be a vehicle for eliminating disparities if used to root out causes of disease, to reprogram the biology of disease and to target the most vulnerable people for early intervention. However, investing in healthy communities and developing social and economic policies that increase opportunities, education and access to quality health care will go a long way toward breaking the cycle of health inequalities. Now is the time for policy-makers to consider undertaking a vigorous debate about how scientific advances could improve the public's health, given the complexities of our health care delivery system and the economic and cultural differences that constitute our nation.

\section{CONCLUSIONS}

The tools of modern science have surprised, intrigued and dazzled our imagination. They have improved the oral health of and quality of life for countless people and communities. Yet, we have learned that oral diseases have no anatomical or disciplinary borders. As we look toward the future of oral health care and research, our best prospects for making the next leap toward solving complex oral diseases-from caries to clefting or from rare craniofacial disorders to oral cancer-are by supporting the best science, embracing new avenues of inquiry and welcoming the expertise of people in other disciplines.

When Congress established NIDCR more than 60 years ago, our nation's oral health was so poor that one could not have anticipated today's remarkable gains. Scientific research will continue to yield exciting technologies and effective treatments. Dental educators and clinicians will need to rise to the challenge of adapting to novel ways of providing care and applying new approaches to solving old problems. Doing so will ensure that our profession is poised to inherit the future.

\section{Acknowledgments}

The authors acknowledge the contributions of Mr. Bob Kuska and Ms. Alison Davis to the manuscript of this article. 


\section{ABBREVIATION KEY}

NIDCR National Institute of Dental and Craniofacial Research

\section{References}

1. Hoffer, E. Reflections on the Human Condition. Titusville, N.J: Hopewell Publications; 2006.

2. Schein, EH.; Kampas, PJ.; Delisi, P.; Sonduck, M. Ken Olsen, the scientist-engineer. In: Schein, EH., editor. DEC Is Dead, Long Live DEC: The Lasting Legacy of Digital Equipment Corporation. San Francisco: Berrett-Koehler; 2003. p. 38

3. Olsen, Ken. [Accessed July 1, 2009] Snopes.com. "www.snopes.com/quotes/kenolsen.asp"

4. Editorials. What's the next big thing? Softpedia; "http://news.softpedia.com/news/What-s-the-Next-Big-Thing-17057.shtml"

5. Oxman AD, Fretheim A, Flottorp S. The OFF theory of research utilization. J Clin Epidemiol. 2005; 58(2):113-116. [PubMed: 15680741]

6. Burt, BA.; Eklund, SA.; Lewis, DW.; Striffler, DF. Dentistry, Dental Practice, and the Community. 4. Philadelphia: Saunders; 1992. p. 301

7. Highfield, R. Future of science: 'we will have the power of the gods.'. Telegraph.co.uk; "www.telegraph.co.uk/scienceandtechnology/science/sciencenews/3311478/Future-of-science-Wewill-have-the-power-of-the-gods.html"

8. The Wong Lab at the UCLA School of Dentistry. Dental Research Institute; "www.hspp.ucla.edu/wonglab/"

9. Plummer, DC.; Cearley, DW.; Smith, DM. Cloud computing confusion leads to opportunity. Gartner Research; ID G00159034.

"www.gartner.com/it/products/research/cloud_computing/cloud_computing.jsp"

10. Kaplan, G. Socioeconomic inequalities in health: what they look like and what can be done about them (edited transcript). Presented at: Health Status Disparities in the United States; April 4, 2007 ; Washington. "http://wilsoncenter.org/events/docs/kaplan1.pdf" 


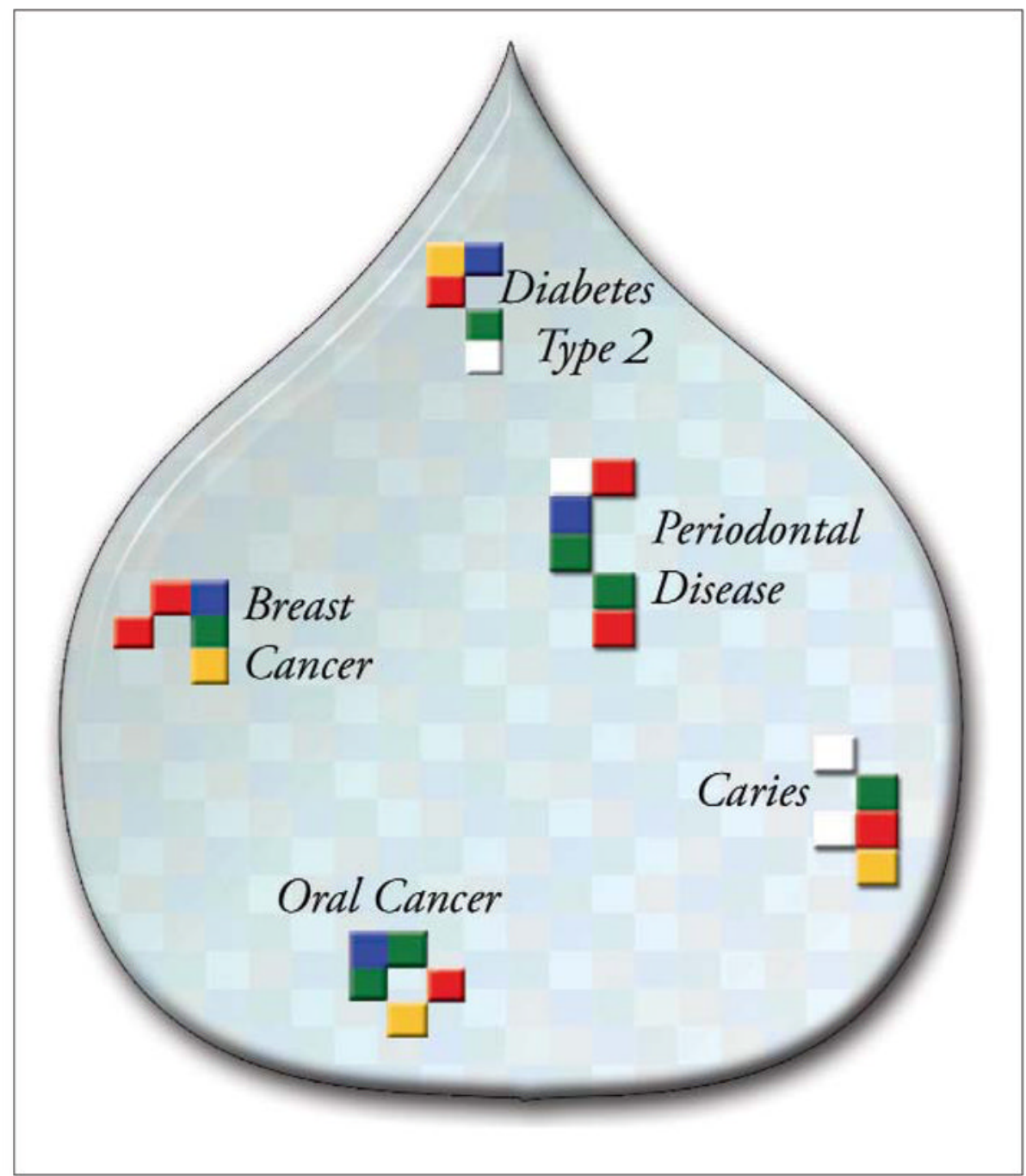

$1 .$.

Envisioned signatures for oral cancer, breast cancer, type 2 diabetes, periodontal disease and caries. Adapted with permission of Wong from The Wong Lab. ${ }^{8}$ 